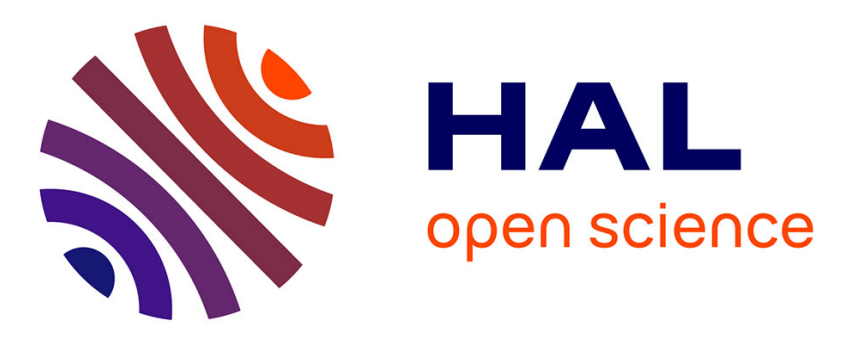

\title{
Relay selection in two-way relaying network with the presence of hardware impairment at relay transceiver
}

Nguyen Hong Nhat, Vo Nguyen Quoc Bao, Linh Trung Nguyen, Merouane Debbah

\section{- To cite this version:}

Nguyen Hong Nhat, Vo Nguyen Quoc Bao, Linh Trung Nguyen, Merouane Debbah. Relay selection in two-way relaying network with the presence of hardware impairment at relay transceiver. International Conference on Advanced Technologies for Communications (ATC 2014), Oct 2014, Hnoi, Vietnam. 10.1109/atc.2014.7043462 . hal-01098956

\section{HAL Id: hal-01098956 https://hal.science/hal-01098956}

Submitted on 17 Jan 2015

HAL is a multi-disciplinary open access archive for the deposit and dissemination of scientific research documents, whether they are published or not. The documents may come from teaching and research institutions in France or abroad, or from public or private research centers.
L'archive ouverte pluridisciplinaire HAL, est destinée au dépôt et à la diffusion de documents scientifiques de niveau recherche, publiés ou non, émanant des établissements d'enseignement et de recherche français ou étrangers, des laboratoires publics ou privés. 


\title{
Relay selection in two-way relaying network with the presence of hardware impairment at relay transceiver
}

\author{
Nguyen Hong Nhat ${ }^{1}$, Vo Nguyen Quoc Bao ${ }^{2}$, Nguyen Linh Trung ${ }^{3}$ and Merouane Debbah. ${ }^{4}$ \\ ${ }^{1}$ Master Student at University of Engineering and Technology, Vietnam National University, Hanoi, Vietnam (e-mail: nhnhat@hue.vnn.vn) \\ ${ }^{2}$ Posts and Telecommunications Institute of Technology, Vietnam (e-mail: baovnq@ptithcm.edu.vn) \\ 3 University of Engineering and Technology, Vietnam National University, Hanoi, Vietnam (e-mail: linhtrung@ vnu.edu.vn) \\ ${ }^{4}$ Alcatel-Lucent Chair on Flexible Radio, SUPELEC, France (e-mail: merouane. debbah@supelec.fr)
}

\begin{abstract}
In this paper, we analyze the performance of twoway amplify-and-forward (AF) relaying with best relay selection scheme in the presence of hardware impairment at the relays. We first obtain the tractable closed-form expressions of the statistical characteristics for end-to-end signal-to-noise ratio (SNR). To reveal significant insights into the system performance, we also computed the asymptotic SEP, which is shown extremely tight in the high SNR regime and low hardware impairments level. Our analysis enables us to evaluate the impact of non-ideal hardware on the performance of two-way relaying networks and highlight as a benchmark for system designer.
\end{abstract}

Index Terms-relay hardware impairment, relay selection, amplify-and-forward

\section{INTRODUCTION}

A two-way AF relaying network has two sources and a single or multiple relays. In the first time slot, two source nodes transmit signal to the relay (for single relay network) or to all relays (for multiple relays network) simutaneously. In single relay network, the relay received the superimposed signal from two source node. In the second time slot, the relay amplifies the received signal and then forward it to two source nodes. The performance of single relay network using AF protocol was discussed in [1], [2].

Many researchs showed that the performance of a wireless network with multiple relays is improved by using relay selection methods. In [3], it presented a Max - Min sum rate selection method based on outage probability. [4] has a algorithm of selecting the relay so that the received SNR is maximized. In [5], the author proposed a Max-Min SNR selection method. In [6], the diversity orders of many the single-relay selection schemes in a two-way wireless relay network is analyzed. A Min-Max criterion for relay selection is introduced in [7], where a relay with a minimizes the maximum SEP of two sources will be selected. The selection scheme in [7] also showed that it has full diversity order of $\mathrm{N}$ if the system has $\mathrm{N}$ relays but it provide better BER than all-participate amplify-and-forward (AP-AF) relaying scheme.

The analyses in above researchs are done with the assumption that the hardware of the relays are perfect. In practice, the hardware is affected by impairments such as IQ imbalance, amplifier non-linearities, and phase noise (see [8]), and the hardware impairments of the relays alway have many effects on wireless communications.

To make the system more realistic, in this paper, we will analyze a relay selection of two-way AF relaying network as proposed in [7] but with the presence of hardware impairment at relaying nodes. Our purpose is the consider of the effects of hardware impairments on relay selection of two-way $\mathrm{AF}$ relaying wireless network.

The paper is orangnized as follows. The system model for considered two-way multiple relays AF network is describled in Section II. In that section we extract the expressions of transmission/received signals at two source nodes and at the relays of network. Then, in section III, we introduce two relay selection amplify-and-forward (RS-AF) scheme: Optimal Relay Selection Amplify-and-Forward (O-RS-AF) and Suboptimal Relay Selection Amplify-and-Forward (S-RS-AF). Our analysis concentrate on the proposed Min-Max selection criterion of S-RS-AF method and the asymptotic SEP is also computed. Numerical results are shown in section IV and finally, section $\mathrm{V}$ is our conclusion.

Notation: $\mathcal{C N}\left(\mu, \sigma^{2}\right)$ is complex Gaussion distribution with mean $\mu$ and variance $\sigma^{2} . \operatorname{Var}\{\},. E\{$.$\} are variance and ex-$ pectation of random variable. $K_{n}($.$) is the n$ th-order modified Bessel function of the second kind .

\section{System AND ChanNEl Model}

Consider two-way relaying network with two source nodes denoted by $S_{1}$ and $S_{2}$ and $N$ relays denoted by $R_{1}, R_{2}, \ldots, R_{N}$. All nodes have single antenna. In the first time slot, both source nodes send information to all relays. Each relay receive the super-position signal from $S_{1}$ and $S_{2}$. In the second time slot, an optimal relay node is selected to forward received signal to two source nodes while other relays keep idle. In this model, we use quasi-static Rayleigh fading channels with additive white Gaussian noise. We assume that all sources or relay nodes know can have perfect channel estimation. The method of selection the relay node as in [7], but this paper research the condition when each relay node suffer the impairment of transceiver hardware.

The symbol transmitted by $S_{i}$ is $s_{i}(i=1,2)$. The signal received at the $k$-th relay in first time slot is: 


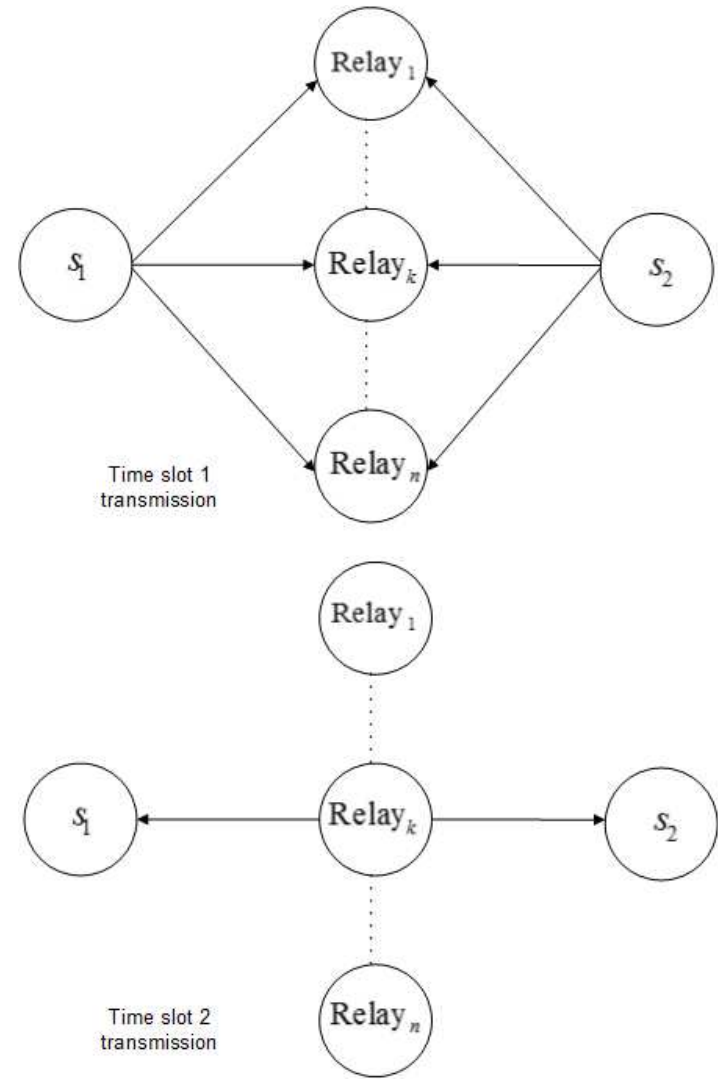

Fig. 1. Relay selection network

$$
y_{r_{k}}=\sqrt{p_{s}} h_{1, r_{k}} s_{1}+\sqrt{p_{s}} h_{2, r_{k}} s_{2}+n_{r_{k}}+\eta_{r_{k}}
$$

where $p_{s}$ is transmit power at source nodes $S_{1}$ and $S_{2}$, $h_{i, r_{k}}(i=1,2 ; k=1,2, . . N)$ are fading coefficient between $S_{i}$ and $R_{k}$ with zero mean and unit variance; $n_{r_{k}}$ is addictive white noise $n_{r_{k}} \sim \mathcal{C N}\left(0, N_{0}\right) ; \eta_{r_{k}}$ is hardware distorsion noise of relay receiver of $R_{k}: \eta_{r_{k}} \sim \mathcal{C N}\left(0, \kappa_{r}^{2}\left(p_{s}\left|h_{1, r_{k}}\right|^{2}+\right.\right.$ $\left.p_{s}\left|h_{2, r_{k}}\right|^{2}\right)$ ) (see A for hardware impairment model).

The relay $R_{k}$ then processes the received signal and forward to two source nodes. The signal generate by the relay is:

$$
x_{r_{k}}=\beta_{k} y_{r_{k}}
$$

where $\beta_{k}$ is amplification factor. It can be expressed as $\beta_{k}=$ $\left(\left(p_{s}\left|h_{1, r_{k}}\right|^{2}+p_{s}\left|h_{2, r_{k}}\right|^{2}\right)\left(1+\kappa_{r}^{2}\right)+N_{0}\right)^{-1 / 2}$.

Denote $\eta_{t_{k}}$ is hardware impairment of transmitter at the relay: $\eta_{t_{k}} \sim \mathcal{C} \mathcal{N}\left(0, \kappa_{t}^{2} p_{r}\right), p_{r}$ is transmit power of the relay.

The relay forward $x_{r_{k}}$ to two source nodes $S_{i}(i=1,2)$, if $y_{i, r_{k}}^{\prime}$ is the signal received at $S_{i}$ from $R_{k}$, it can be written as

$$
y_{i, r_{k}}^{\prime}=\sqrt{p_{r}} h_{i, r_{k}} x_{r_{k}}+h_{i, r_{k}} \eta_{t_{k}}+n_{i, r_{k}}, \quad i=1,2
$$

Denote $\quad \alpha_{k}=\sqrt{p_{s} p_{r}} \beta_{k} h_{1, r_{k}} h_{2, r_{k}}, \quad \omega_{1, r_{k}}=$ $\sqrt{p_{r}} \beta_{k} h_{1, r_{k}}\left(n_{r_{k}}+\eta_{r_{k}}\right)+h_{1, r_{k}} \eta_{t_{k}}+n_{1, r_{k}}, \omega_{2, r_{k}}=$ $\sqrt{p_{r}} \beta_{k} h_{2, r_{k}}\left(n_{r_{k}}+\eta_{r_{k}}\right)+h_{2, r_{k}} \eta_{t_{k}}+n_{2, r_{k}}$. Combine (1), (2), (3) and after subtracting its own information, each source node $S_{i}(i=1,2)$ we have the received signal of each source node

$$
y_{1, r_{k}}=\alpha_{k} s_{2}+\omega_{1, r_{k}}, y_{2, r_{k}}=\alpha_{k} s_{1}+\omega_{2, r_{k}}
$$

The recover signal can be archived by using Maximum likehood detector. Two relay selection schemes are discussed in the next section.

\section{SEP ANALYSIS}

We use relay selection methods to choose one best relay and then transmit the super-position signal to two sources. We have two relaying selection methods, as proposed in [7]:

1). Optimal Relay Selection Amplify-and-Forward (O-RSAF) scheme: The selected relay denoted by $\mathcal{R}$ which has the minimum SEP from both source:

$$
\begin{gathered}
\mathcal{R}=\min _{k}\left\{S E P_{1, r_{k}}\left(\gamma_{1, r_{k}} \mid h_{1, r_{k}}, h_{2, r_{k}}\right),\right. \\
\left.+S E P_{2, r_{k}}\left(\gamma_{2, r_{k}} \mid h_{1, r_{k}}, h_{2, r_{k}}\right)\right\}
\end{gathered}
$$

$S E P_{1, r_{k}}\left(\gamma_{1, r_{k}} \mid h_{1, r_{k}}, h_{2, r_{k}}\right), \quad i=1,2$ are SEP at source nodes $S_{i}$ from $k$-th relay with channel coefficient $h_{1, r_{k}}, h_{2, r_{k}}$. According to [7] we have $S E P_{i, r_{k}}\left(\gamma_{i, r_{k}} \mid h_{1, r_{k}}, h_{2, r_{k}}\right)=$ $Q\left(\sqrt{c \gamma_{i, r_{k}}}\right)$, which $Q(x)=\frac{1}{\sqrt{2 \pi}} \int_{x}^{\infty} \exp \left(-t^{2} / 2\right) d t$ is $Q$ is $Q($.) function and if we use BPSK modulation we have $c=2$. $\gamma_{i, r_{k}}$ is SNR at source nodes:

$$
\gamma_{i, r_{k}}=\frac{\left|\alpha_{k}\right|^{2}}{\operatorname{Var}\left\{\omega_{i, r_{k}}\right\}}
$$

2). Sub-Optimal Relay Selection Amplify-and-Forward (SRS-AF) scheme: It is very difficult to analyze (4), so a new Min-Max selection criteria is proposed. The Min-Max selection can be describles by following equation

$$
\begin{gathered}
\mathcal{R}=\operatorname{minmax}_{k}\left\{S E P_{1, r_{k}}\left(\gamma_{1, r_{k}} \mid h_{1, r_{k}}, h_{2, r_{k}}\right),\right. \\
\left.\operatorname{SEP}_{2, r_{k}}\left(\gamma_{2, r_{k}} \mid h_{1, r_{k}}, h_{2, r_{k}}\right)\right\}
\end{gathered}
$$

Equation (5) is equivalent to estimate the SNR equation

$$
\gamma_{R}=\operatorname{maxmin}_{k}\left\{\gamma_{1, r_{k}}, \gamma_{2, r_{k}}\right\}, \quad k=1, \ldots, N
$$

Theorem 1. Define $\psi_{r} \triangleq \frac{p_{r}}{N_{0}\left(1+\kappa_{r}^{2}\right)}, \quad \psi_{s} \triangleq \frac{p_{s}}{N_{0}} \frac{1}{(1+\lambda)\left(1+\kappa_{t}^{2}\right)}$ with $\lambda \triangleq \frac{p_{s}\left(1+\kappa_{r}^{2}\right)}{p_{r}\left(1+\kappa_{t}^{2}\right)}$. In high SNR regime and low impairments level, the asymptotic average SEP of this S-RS-AF scheme is:

$$
S E P_{R S}=\frac{(2 N-1) ! !}{2}\left(\frac{\psi}{c}\right)^{N}
$$

where $(2 n-1) ! ! \triangleq \prod_{k=1}^{n}(2 k-1)=\frac{(2 n-1) !}{n ! 2^{n}}$ and $\psi=$ $2\left(\psi_{r}^{-1}+\psi_{s}^{-1}\right)$.

Proof: First, we compute the PDF and CDF of $\gamma_{R}$ from equation (6).

$\gamma_{1, r_{k}}$ and $\gamma_{2, r_{k}}$ has the same PDF and CDF, denoted by $f_{\gamma_{k}}(x)$ and $F_{\gamma_{k}}(x)$.

$$
\gamma_{1, r_{k}}=\frac{\left|\alpha_{k}\right|^{2}}{\operatorname{Var}\left\{\omega_{1, r_{k}}\right\}}
$$




$$
\begin{array}{rcc}
\operatorname{Var}\left\{\omega_{1, r_{k}}\right\} & = & p_{r} \beta_{k}^{2} N_{0}\left|h_{1, r_{k}}\right|^{2} \\
& + & p_{r} \beta_{k}^{2}\left|h_{1, r_{k}}\right|^{2} \kappa_{r}^{2}\left(p_{s}\left|h_{1, r_{k}}\right|^{2}+p_{s}\left|h_{2, r_{k}}\right|^{2}\right) \\
& + & \left|h_{1, r_{k}}\right|^{2} \kappa_{t}^{2} p_{r}+N_{0}
\end{array}
$$

After some computational steps, we have:

$$
\gamma_{1, r_{k}}=\frac{\frac{p_{s}}{N_{0}} \frac{p_{r}}{N_{0}}\left|h_{1, r_{k}}\right|^{2}\left|h_{2, r_{k}}\right|^{2}}{\left(\frac{p_{r}\left(1+\kappa_{t}^{2}\right)+p_{s}\left(1+\kappa_{r}^{2}\right)}{N_{0}}\right)\left|h_{1, r_{k}}\right|^{2}+\frac{p_{s}\left(1+\kappa_{r}^{2}\right)}{N_{0}}\left|h_{2, r_{k}}\right|^{2}+\xi}
$$

$$
(10)_{\gamma k}(x)=2 \beta_{1} \beta_{2} x e^{-x\left(\beta_{1}+\beta_{2}\right)} \times\left[\left(\frac{\beta_{1}+\beta_{2}}{\sqrt{\beta_{1} \beta_{2}}}\right) \times K_{1}\left(2 x \sqrt{\beta_{1} \beta_{2}}\right)\right.
$$

where

$$
\begin{aligned}
\xi= & 1+\frac{\left|h_{1, r_{k}}\right|^{2}\left|h_{2, r_{k}}\right|^{2}\left(p_{r} p_{s} \kappa_{r}^{2}+p_{s} p_{r}\left(1+\kappa_{r}^{2}\right) \kappa_{t}^{2}\right)}{N_{0}^{2}} \\
& +\frac{\left|h_{1, r_{k}}\right|^{4}\left(p_{r} p_{s} \kappa_{r}^{2}+p_{s} p_{r}\left(1+\kappa_{r}^{2}\right) \kappa_{t}^{2}\right)}{N_{0}^{2}}
\end{aligned}
$$

Define $\psi_{r} \triangleq \frac{p_{r}}{N_{0}\left(1+\kappa_{r}^{2}\right)}, \quad \psi_{s} \triangleq \frac{p_{s}}{N_{0}} \frac{1}{(1+\lambda)\left(1+\kappa_{t}^{2}\right)}$ with $\lambda \triangleq$ $\frac{p_{s}\left(1+\kappa_{r}^{2}\right)}{p_{r}\left(1+\kappa_{t}^{2}\right)}$ we can rewrite (10) as:

$$
\begin{aligned}
\gamma_{1, r_{k}} & =\frac{\psi_{r} \psi_{s}\left|h_{1, r_{k}}\right|^{2}\left|h_{2, r_{k}}\right|^{2}}{\psi_{r}\left|h_{1, r_{k}}\right|^{2}+\psi_{s}\left|h_{2, r_{k}}\right|^{2}+\xi^{\prime}} \\
& \approx \frac{\psi_{r} \psi_{s}\left|h_{1, r_{k}}\right|^{2}\left|h_{2, r_{k}}\right|^{2}}{\psi_{r}\left|h_{1, r_{k}}\right|^{2}+\psi_{s}\left|h_{2, r_{k}}\right|^{2}}
\end{aligned}
$$

where $\xi^{\prime} \rightarrow 0$ when SNR is high and $\kappa_{r}, \kappa_{t} \rightarrow 0$.

(The remain proof of this theorem can refer to [7]).

Define $\gamma_{k}^{\text {min }} \triangleq \min \left\{\gamma_{1, r_{k}}, \gamma_{2, r_{k}}\right\}, f_{\gamma_{k}^{\text {min }}}(x)$ and $F_{\gamma_{k}^{\text {min }}}(x)$ are PDF and CDF function, respectively. Using order statistics as in [9], we can caculate PDF of $\gamma_{R}$ :

$$
\begin{gathered}
f_{\gamma_{R}}(x)=N f_{\gamma_{k}^{\min }}(x) F_{\gamma_{k}^{\text {min }}}^{N-1}(x) \\
F_{\gamma_{R}}(x)=F_{\gamma_{k}^{\min }}^{N}(x)
\end{gathered}
$$

where $f_{\gamma_{k}^{\min }}(x)=2 f_{\gamma_{k}}(x)\left(1-F_{\gamma_{k}}(x)\right), \quad F_{\gamma_{k}^{\min }}(x)=1-$ $\left(1-F_{\gamma_{k}}(x)\right)^{2}$

From equations (13) and (14):

$$
f_{\gamma_{R}}(x)=2 N f_{\gamma_{k}}(x)\left(1-F_{\gamma_{k}}(x)\right)\left[1-\left(1-F_{\gamma_{k}}(x)\right)^{2}\right]^{N-1}
$$

From (12), let $X_{1} \triangleq \psi_{s}\left|h_{1, r_{k}}\right|^{2}, X_{2} \triangleq \psi_{r}\left|h_{2, r_{k}}\right|^{2}$ :

$$
\gamma_{1, r_{k}}=\frac{1}{\frac{1}{\psi_{s}\left|h_{1, r_{k}}\right|^{2}}+\frac{1}{\psi_{r}\left|h_{2, r_{k}}\right|^{2}}}=\frac{1}{2} \frac{2}{\frac{1}{X_{1}}+\frac{1}{X_{2}}}
$$

$\frac{2}{\frac{1}{X_{1}}+\frac{1}{X_{2}}}$ is harmonic mean of two random variables has exponential distributed function:

$$
p_{X_{i}}(x)=\beta_{i} e^{-\beta_{i} x} U(x)
$$

where $\beta_{1}=1 / \psi_{s}, \beta_{2}=1 / \psi_{r} . U(x)$ is unit step function. According [10] $X=\mu_{H}\left(X_{1}, X_{2}\right)=\frac{2}{\frac{1}{X_{1}}+\frac{1}{X_{2}}}$ has PDF:

$$
\begin{aligned}
f_{X}(x)= & \frac{1}{2} \beta_{1} \beta_{2} x e^{-\frac{x\left(\beta_{1}+\beta_{2}\right)}{2}}\left[\left(\frac{\beta_{1}+\beta_{2}}{\sqrt{\beta_{1} \beta_{2}}}\right) \times K_{1}\left(x \sqrt{\beta_{1} \beta_{2}}\right)\right. \\
& \left.+2 K_{0}\left(x \sqrt{\beta_{1} \beta_{2}}\right)\right] U(x)
\end{aligned}
$$

$K_{0}(),. K_{1}($.$) are zeroth-order and first-order modified$ Bessel functions of the second kind..

Let $X^{\prime}=\frac{1}{2} \mu_{H}\left(X_{1}, X_{2}\right)=\frac{1}{2} X$. Subtituted $x=2 x^{\prime}$ in (16) , we have PDF of $X^{\prime}$ (notice that $d x=2 d x^{\prime}$ ):

$$
\left.+2 K_{0}\left(2 x \sqrt{\beta_{1} \beta_{2}}\right)\right] U(x)
$$

Because $\beta_{1}+\beta_{2}=\psi_{r}^{-1}+\psi_{s}^{-1}, \quad \beta_{1} \beta_{2}=\frac{1}{\psi_{r} \psi_{s}}, \frac{\beta_{1}+\beta_{2}}{\sqrt{\beta_{1} \beta_{2}}}=$ $\frac{\psi_{r}+\psi_{s}}{\sqrt{\psi_{r} \psi_{s}}}$, so:

$$
\begin{aligned}
f_{\gamma_{k}}(x)= & \frac{2 x e^{-x\left(\psi_{r}^{-1}+\psi_{s}^{-1}\right)}}{\psi_{r} \psi_{s}} \times\left[\frac{\psi_{r}+\psi_{s}}{\sqrt{\psi_{r} \psi_{s}}} \times K_{1}\left(\frac{2 x}{\sqrt{\psi_{r} \psi_{s}}}\right)\right. \\
& \left.+2 K_{0}\left(\frac{2 x}{\sqrt{\psi_{r} \psi_{s}}}\right)\right] U(x)
\end{aligned}
$$

And we also have $K_{1}(z) \rightarrow 1 / z$ when $z \rightarrow 0, K_{0}(z) \rightarrow 0$ when $z \rightarrow 0$. So when SNR is high:

$$
\begin{aligned}
f_{\gamma_{k}}= & \frac{2 x}{\psi_{r} \psi_{s}} e^{-x\left(\psi_{r}^{-1}+\psi_{s}^{-1}\right)} \times \frac{\psi_{r}+\psi_{s}}{\sqrt{\psi_{r} \psi_{s}}} \times \frac{\sqrt{\psi_{r} \psi_{s}}}{2 x} \\
f_{\gamma_{k}} & =\frac{\psi_{r}+\psi_{s}}{\psi_{r} \psi_{s}} e^{-x\left(\psi_{r}^{-1}+\psi_{s}^{-1}\right)} \\
& =\left(\psi_{r}^{-1}+\psi_{s}^{-1}\right) e^{-x\left(\psi_{r}^{-1}+\psi_{s}^{-1}\right)}=\frac{\psi}{2} e^{-\frac{\psi}{2} x}
\end{aligned}
$$

where $\psi=2\left(\psi_{r}^{-1}+\psi_{s}^{-1}\right)$.

And the CDF is:

$$
F_{\gamma_{k}}(x)=1-e^{-\frac{\psi}{2} x}
$$

Replace $f_{\gamma_{k}}(x), F_{\gamma_{k}}(x)$ from (17),(18) to (15), we have:

$$
f_{\gamma_{R}}(x)=N \psi e^{-\psi x}\left(1-e^{-\psi x}\right)^{N-1}
$$

The fact that $\left(1-e^{-x}\right) \approx x$ when $x \rightarrow 0$ (Taylor series), so when SNR goes high $(\psi \rightarrow 0)$, from (14) and (18):

$F_{\gamma_{R}}(x)=F_{\gamma_{k}^{\min }}^{N}(x)=\left[1-\left(1-F_{\gamma_{k}}(x)\right)^{2}\right]^{N}=\left(1-e^{-\psi x}\right)^{N} \rightarrow(\psi x)^{N}$

The average SEP can be computed with equation:

$$
S E P_{R S}=\mathbb{E}\left[S E P\left(\gamma_{R} \mid h_{1}, h_{2}\right)\right]=\mathbb{E}\left[Q\left(\sqrt{c \gamma_{R}}\right)\right]
$$

$c$ is a constant determined by the modulation format, if use BPSK modulation then $c=2$.

If $X$ is a random variables with $X \sim \mathcal{N}(0,1)$, the average SEP can be rewritten as: 


$$
\begin{aligned}
S E P & =P\left\{X>\sqrt{c \gamma_{R}}\right\}=P\left\{\gamma_{R}<\frac{X^{2}}{c}\right\} \\
& =\mathbb{E}\left[F_{\gamma_{R}}\left(\frac{X^{2}}{c}\right)\right]=\int_{0}^{\infty} F_{\gamma_{R}}\left(\frac{X^{2}}{c}\right) F_{X}(x) d x
\end{aligned}
$$

From (19) and $X \sim \mathcal{N}(0,1)$ we have:

$$
S E P_{R S}=\frac{1}{\sqrt{2 \pi}}\left(\frac{\psi}{c}\right)^{N} \int_{0}^{\infty} x^{2 N} e^{-\frac{x^{2}}{2}} d x
$$

Using the result from [11]: $\int_{0}^{\infty} t^{2 n} e^{-k t^{2}} d t=$ $\frac{(2 n-1) ! !}{2(2 k)^{n}} \sqrt{\frac{\pi}{k}}$ with $(2 n-1) ! ! \triangleq \prod_{k=1}^{n}(2 k-1)=\frac{(2 n-1) !}{n ! 2^{n}}$, replace $k=1 / 2$ we have:

$$
S E P_{R S}=\frac{(2 N-1) ! !}{2}\left(\frac{\psi}{c}\right)^{N}
$$

Noticed that $\psi=2\left(\psi_{r}^{-1}+\psi_{s}^{-1}\right)<1$ so from SEP at (20) we see that the diversity of this S-RS-AF scheme is $\mathrm{N}$ when using $\mathrm{N}$ relay nodes.

We have following corollary:

Corollary 2. a)If the relay transceiver is ideal, or $\kappa_{r}=\kappa_{t}=$ $0 \Rightarrow \psi_{r}=\frac{p_{r}}{N_{0}}, \psi_{s}=\frac{p_{s}}{N_{0}} \frac{1}{(1+\lambda)}, \lambda=\frac{p_{s}}{p_{r}}$, we have the same result of $\psi_{r}, \psi_{t}, \lambda$ and the equation (12) as in [7].

b)Denote $S N R=p_{s} / N_{0}$ is signal-noise ratio of source, we have $N_{0} / p_{s}=1 / S N R, N_{0} / p_{r}=\lambda\left(1+\kappa_{t}^{2}\right) /\left(1+\kappa_{r}^{2}\right) * 1 / S N R$ and $\psi=2\left(\psi_{r}^{-1}+\psi_{s}^{-1}\right)=2 \frac{1}{S N R}(1+2 \lambda)\left(1+\kappa_{t}^{2}\right)$, (20) become:

$S E P_{R S}=\frac{(2 N-1) ! !}{2}\left(\frac{\psi}{c}\right)^{N}=\frac{(2 N-1) !}{N ! 2 c^{N}} \frac{(1+2 \lambda)^{N}}{S N R^{N}}\left(1+\kappa_{t}^{2}\right)$

\section{Numerical Results AND Discussion}

In this simulation, we assume both source nodes have the SNR. BPSK modulation is used and all channels are symetric Rayleigh fading channels.

Total energy of two source nodes $S_{1}, S_{2}$ and all relays $R_{k}(k=1, . ., N)$ are $p$. The number $N=1,2,3,4$ relay nodes is used in these simulations.

Noise variance off all channels are $N_{0}$.

With Relay Selection Scheme in this paper, we only has a single relay work to forward signal to two source nodes. In this simulation, $p_{s}=p_{r}=1$.

As in [6], the system is outage if the hardware impairment coefficient is over 0.2 , so all the simulation we use the low hardware impairment levels to satify the assumption of this research.

Firgure 2 is the result average SEP of O-RS-AF and S-RS$\mathrm{AF}$ with the hardware impairment level is $\kappa_{r}=0.01, \kappa_{t}=$ 0.03 , we can see that the O-RS-AF method and S-RS-AF has the same performance result. With diffrent levels of impairments we alway has the same result of O-RS-AF and S-RS-AF but because the limit length of this paper we don't include them in. From that figure we see that the performance

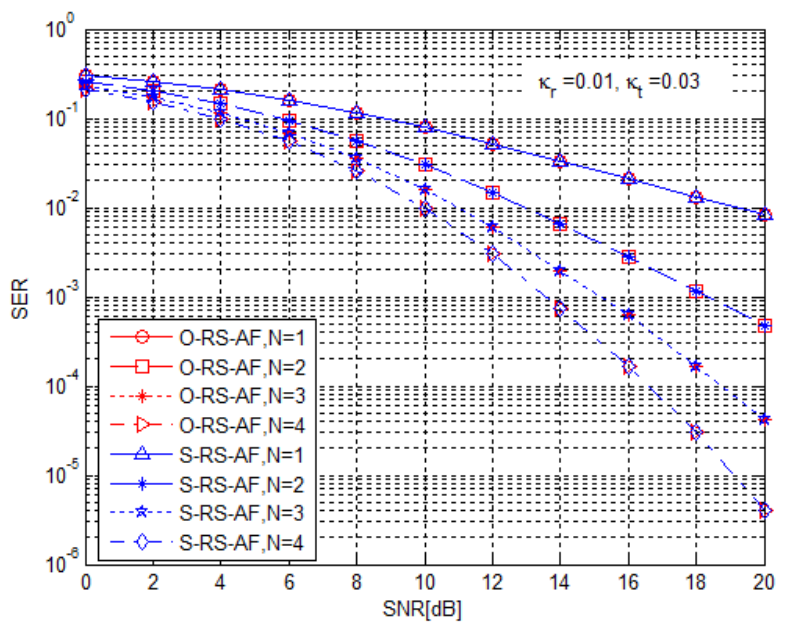

Fig. 2. Compare simulated SEP performance between optimal and suboptimal relay selection methods when $\mathrm{N}=1 ; 2 ; 3 ; 4$.

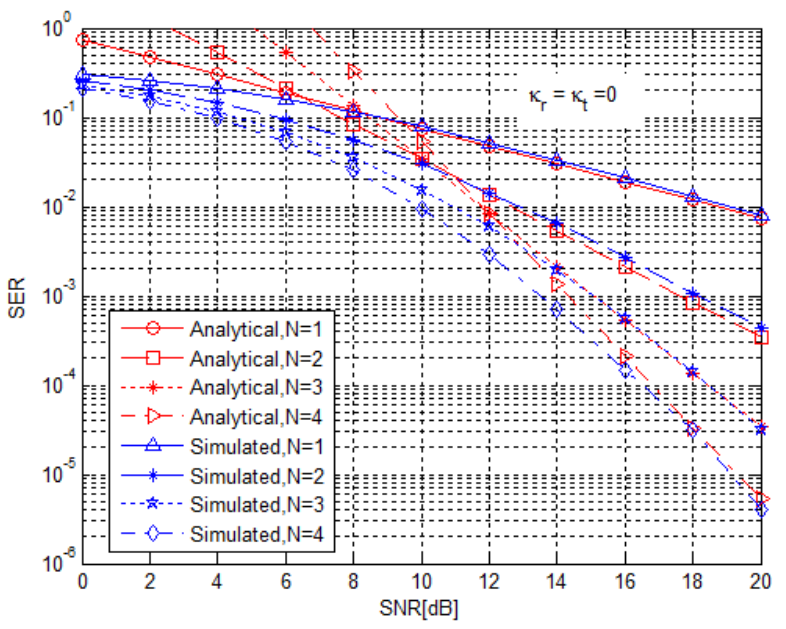

Fig. 3. Compare Analytical and Simulated SEP by the proposed S-RS-AF scheme when the relay hardware is ideal $\kappa_{r}=\kappa_{t}=0$ and $\mathrm{N}=1 ; 2 ; 3 ; 4$



Fig. 4. Compare Analytical and Simulated SEP by the proposed S-RS-AF scheme, where $\kappa_{r}=\kappa_{t}=0.02$ and $\mathrm{N}=1 ; 2 ; 3 ; 4$ 


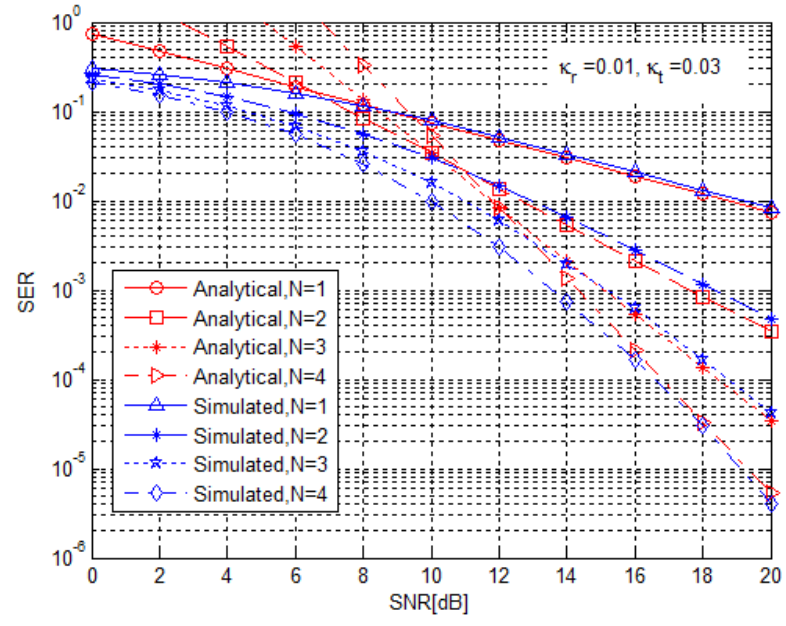

Fig. 5. Compare Analytical and Simulated SER by the proposed S-RS-AF scheme, where $\kappa_{r}=0.01, \kappa_{t}=0.03$ and $\mathrm{N}=1 ; 2 ; 3 ; 4$

is improved when SNR goes high and when number of the relays increase.

Figure 3 compare the simulated SEP using S-RS-AF with the asymptotic analytical SEP using theorem (1), the impairment level is $\kappa_{r}=\kappa_{t}=0$. This occurs when the hardware is ideal and the simulation has the same result as [7].

With $\kappa_{r}=\kappa_{t}=0.02$ we have the comprarision in figure 4 . Figure 5 demonstrates another case where the relay hardware isn't symetric, $\kappa_{r}=0.01, \kappa_{t}=0.03$. Figure 3, 4 and 5 show that with high SNR and low hardware impairment level of relay transceiver, the analytical SEP is converged to the simulated result.

\section{CONCLUSIONS}

In this paper, we research the Relay Selection Method of two-way relaying network. But don't like in [7], this paper compute the effect of relay hardware tranceiver and also find in which conditions the asymptotic SEP equation is still can be used.

This paper show that, we can use the Min-Max Relay Selection method as proposed in [7] even when it has the presence of hardware impairment of relays. The result of theorem (1) is more general than the asymptotic result in [7], because the parameter of hardware impairment level is considered. When we use ideal relay hardware, the result of theorem (1) has the same analytical result of [7].

\section{REFERENCES}

[1] P. Popovski and H. Yomo, "Wireless network coding by amplify-andforward for bi-directional traffic flows," Communications Letters, IEEE., vol. 11, pp. 16-18, 2007.

[2] B. Raymond H. Y. Louie; Yonghui Li ; Vucetic, "Practical physical layer network coding for two-way relay channels: performance analysis and comparison," Wireless Communications, IEEE Transactions., vol. 9, pp. $764-777,2010$.

[3] X. J. Zhang and Y. Gong, "Adaptive power allocation in two-way amplify-and-forward relay networks," in Proc. IEEE, 2009, pp. 1 - 5 .

[4] Y. Zhao, R.Adve, and T. Lim, "Symbol error rate of selection amplifyand-forward relay systems," Communications Letters, IEEE, vol. 10, pp. 757-759, 2006.

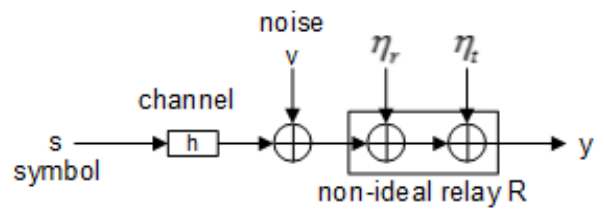

Fig. 6. Hardware impairment model

[5] Y. Jing, "A relay selection scheme for two-way amplify-and-forward relay networks," in International Conference on Wireless Communications and Signal Processing, 2009.

[6] H. X. Nguyen, H. H. Nguyen, and T. Le-Ngoc, "Diversity analysis of relay selection schemes for two-way wireless relay networks," Wireless Personal Communications., vol. 59, pp. 173-189, 2011.

[7] L. Song, "Relay selection for two-way relaying with amplify-andforward protocols," Vehicular Technology, IEEE Transactions., vol. 60, pp. 1954 - 1959, 2011.

[8] M. Matthaiou, A. Papadogiannis, E. Bjornson, and M. Debbah, "Twoway relaying under the presence of relay transceiver hardware impairments," Communications Letters, IEEE., vol. 17, pp. 1136 - 1139, 2013.

[9] H. A. David, Order Statistics. Wiley, 1970.

[10] M. O. Hasna and M. S. Alouini., "End-to-end performance of transmission systems with relays over rayleigh-fading channels," IEEE Trans Wireless Commun., vol. 2, pp. 1126-1131, 2003.

[11] I. S. Gradshteyn and I. M. Ryzhik, Table of Integrals, Series, and Products, A. Jeffrey, Ed. Academic Press, 1994.

[12] T. Schenk, RF Imperfections in High-rate Wireless Systems: Impact and Digital Compensation. Springer, 2008.

\section{APPENDIX A}

TRANSCEIVER HARDWARE IMPAIRMENT MODEL

Consider an information symbol $s \in \mathbb{C}$ is transmited over a wireless channel $h \in \mathbb{C}$ with additive noise $v \in \mathbb{C}$. The received signal is modeled as $y=h s+v$. Because of impairments, the transceivers make: i) the received signal distort during reception processing. ii) generate mismatch between the intented symbol $s$ and accually signal emitted. From [8], [12], the generalized impairment model can be with distortion noises and the received signal is:

$$
y=h\left(s+\eta_{t}\right)+\eta_{r}+v
$$

while $\eta_{t}, \eta_{r}$ are distortion noises from impairments of the transceiver.

And we also have $\eta_{t}, \eta_{r}$ ara Gaussian distribution

$$
\eta_{t} \sim \mathcal{C N}\left(0, \kappa_{t}^{2} P\right), \eta_{r} \sim \mathcal{C N}\left(0, \kappa_{r}^{2} P|h|^{2}\right)
$$

$P$ is average symboy power $P=\mathbb{E}_{s}\left\{|s|^{2}\right\} . \kappa_{t}, \kappa_{r} \geq 0$ are impairment level of transmiter and receiver hardware. When $\kappa_{t}=\kappa_{r}=0$ (it mean ideal hardware), (23) reduce to classical form of received signal $y=h s+v$. 